\title{
A COMPARATIVE STUDY ON CONVENTIONAL AND AUTOMATED BLOOD CULTURE IN THE EARLY DETECTION OF BACTERIAL PATHOGENS
}

\author{
Lekshmi L. Rajan1, B. Jayalekha², Sreekumary P. K3, Harikumar $S^{4}$ \\ ${ }^{1}$ Assistant Professor, Department of Transfusion Medicine, Government Medical College, Kottayam, Kerala. \\ 2 Professor and HOD (Rtd.), Department of Microbiology, Government Medical College, Kottayam, Kerala. \\ ${ }^{3}$ Additional Professor, Department of Microbiology, Government Medical College, Kottayam, Kerala. \\ ${ }^{4}$ DPM, NHM, Kollam, Kerala.
}

\section{ABSTRACT}

\section{BACKGROUND}

Blood culture is a critical tool in the microbiological laboratory, which detects and identifies microorganisms in the blood along with antibiotic susceptibility and has considerable prognostic significance by suggesting appropriate antibiotic therapy. Automated blood culture systems are replacing conventional methods and must be comparatively studied to elucidate the benefits and advantages. The present study compares a recently installed BacT/ALERT automated system with the conventional method with regard to rate of detection and time to detection of microorganisms. This prospective observational study was conducted in a Medical College, a tertiary care centre in Kerala among patients with suspected bacteraemia and sepsis.

\section{MATERIALS AND METHODS}

Two samples were drawn from each of the 248 subjects and subjected to conventional and automated blood cultures using standardised techniques. The rate of detection and the time to detection of microorganisms were compared.

\section{RESULTS}

Overall, $27.8 \%$ and $21.3 \%$ of the samples showed positive growths by the automated and conventional methods. The automated system detected $96.9 \%$ of the single isolates compared to $80 \%$ for the conventional method (p value $<0.01$ ). The automated system detected $72.5 \%$ of the cases within 24 hours and $98.6 \%$ cases within 48 hours. In comparison, the conventional system detected none within 24 hours and only $34 \%$ within 48 hours. The mean time to detection by the automated method was 22.8 , 18.24 and 23 hours for Gram positive bacteria, Gram negative bacteria and fungi respectively.

\section{CONCLUSION}

Automated blood culture systems are a reliable alternative to conventional blood culture systems with better performance in rate of detection and time to detection of micro-organisms in our settings. Early availability of results facilitates the timely initiation of appropriate antibiotic therapy.

\section{KEYWORDS}

Blood Culture, Automated Blood Culture Systems, Bacteraemia, Sepsis.

HOW TO CITE THIS ARTICLE: Rajan LL, Jayalekha B, Sreekumary PK, et al. A comparative study on conventional and automated blood culture in the early detection of bacterial pathogens. J. Evolution Med. Dent. Sci. 2017;6(31):2502-2506, DOI: $10.14260 /$ Jemds/2017/542

\section{BACKGROUND}

Blood culture is a means of detecting the presence of living organisms in the blood through a microbiological culture. Blood culture represents a critical tool for the healthcare professional, as blood is normally sterile and a positive blood culture result is highly significant and it indicates a lifethreatening situation, which requires immediate action. A positive blood culture can suggest a definitive diagnosis and enables targeted therapy against the specific organism(s) in question and provide prognostic value. Detection of bacteraemia and fungaemia followed by antimicrobial

Financial or Other, Competing Interest: None.

Submission 11-03-2017, Peer Review 04-04-2017,

Acceptance 10-04-2017, Published 17-04-2017.

Corresponding Author:

Dr. Lekshmi L. Rajan,

Assistant Professor,

Department of Transfusion Medicine \& Immunohematology,

Government Medical College,

Kottayam, Kerala.

E-mail: lekshmraj@gmail.com

DOI: $10.14260 /$ jemds $/ 2017 / 542$ sensitivity testing is an important function of clinical microbiology laboratories, as it helps in initiating appropriate and effective antimicrobial treatment for a potentially lifethreatening condition. This demands an effective use of all available methods for the earliest recovery of microorganisms causing blood infections, which includes conventional methods and automated blood culture systems. Conventional blood culture methods have given way to automated and computerised methods in most developed countries, while developing countries like India are in various stages of upgradation. Technological advances have resulted in the availability of different systems, each claiming to be superior to others in different aspects. $1,2,3,4$

Automated systems, which perform well in developed countries may not achieve the same results in the Indian context. In this study, an automated blood culture system that has been installed in a tertiary care centre in Kerala is being compared with the conventional blood culture system to understand the additional benefits obtained if any with regard to rate and time of detection of micro-organisms, thus resulting in better clinical outcome. 


\section{MATERIALS AND METHODS}

\section{Study Design and Setting}

The study has a prospective observational design. Sample size was calculated as 240 . The sample size was estimated based on average $18 \%$ positivity among suspected sepsis cases with precision limits of $6 \%$ and $30 \%$ and an alpha error of 5. Assuming a $10 \%$ dropout, the sample size is reestimated to 237 which is further rounded off to 240 .

Patients admitted with fever and/or sepsis in Medicine, Paediatrics and Infectious Diseases Department was included. Extremely low birth weight infants and haemodynamically unstable patients were excluded from the study.

\section{Study Procedure}

The study commenced after acquiring written permission from the Institutional Ethics Committee. Blood samples were collected after obtaining informed consent. Blood was collected under strict aseptic precautions. After locating a suitable vein the site was disinfected with $70 \%$ ethanol, then $1 \%$ povidone-iodine and again ethanol. Blood was then withdrawn using sterile needle and syringe. The syringe was replaced with fresh sterile needle and then inoculated into the bottle. Two separate samples were taken from the same patient within 1 - 3 hours interval. Most of the samples were collected before giving antibiotics.

One sample was inoculated into conventional blood culture bottle in broth with 1:10 dilution and other into automated blood culture bottle. Second sample was inoculated into automated blood culture bottle (AutoBCS) Bact/ALERT system.

The medium used for conventional blood culture system is Brain Heart infusion broth with $0.05 \%$ Polyanethol sulfonate. Conventional blood culture bottles were incubated at $37^{\circ} \mathrm{C}$ for six hours. Subculture was done after six hours and then on daily basis to Blood agar and MacConkey agar for 10 days. Gram staining was also done at the time of subculture. Direct antibiotic sensitivity was done based on Gram reaction. If there was no growth obtained after regular repeated subculture for 10 days, it was reported as 'Sterile after 10 days of incubation.' If the growth was obtained, colonies were identified by Gram staining along with standard biochemical tests and appropriate antibiograms on Mueller-Hinton agar for each isolate.

If any growth was detected on Automated Blood Culture Systems (AutoBCS), a direct Gram staining of blood culture media was performed along with direct biochemical tests and direct antibiotic sensitivity tests for early reporting. Subculture was done on Blood agar and MacConkey agar, colonies identified and antibiotic sensitivity was done for each isolate. If no growth detected, it was reported as 'Sterile after 10 days of incubation.' In addition, a terminal subculture was done from the AutoBCS bottles to detect any false negatives.

The results of the blood culture were entered in EpiData version 3.1 and analysed using EpiInfo software version 3.5.2. Descriptive analysis was done according to the age, sex and provisional diagnosis of the subjects. Further analysis included a comparison of detection rates and time taken for detection between conventional and automated methods of blood culture. Cross tabulation was done for the rates of detection as per the method of blood culture. McNemar's Chi Square test was done to compare the rate of detection between the two methods. Comparison between conventional and automated methods was also done by calculating the cumulative percentage of rates of detection on different days.

\section{RESULTS}

There were a total of 248 participants in the study from whom two samples of blood was collected for inoculation into conventional and AutoBCS bottles.

The subjects ranged from age of one day to 81 years. Male subjects accounted for $58.9 \%$ and females $41.1 \%$. Neonates and infants less than one year constitute one-fifth of the study sample.

55.7 percent of the study subjects were admitted in the General Medicine ward or Intensive Care Units, while 36.7 percent was admitted in the Paediatric Department. Patients admitted in the Infectious Diseases Department constituted the rest. Almost half the subjects had a provisional diagnosis of sepsis, while 37.5 percent fever. Urinary tract infections, Pneumonia and Meningitis constituted rest of the study subjects.

Among 248 sample pairs, 71 (28.6\%) had positive blood culture results either by conventional, automated or both blood culture methods. Single isolates were found in 65 (26.2\%) sample pairs. Among the six samples which gave a mixed growth five were detected by the AutoBCS, while one sample gave a mixed growth in both methods.

Among the 71 samples with positive growth the automated system detected 69 (97.2\%), while conventional detected 53 (74.7\%) (Refer Table No. 1). Among the positive samples $18(25.4 \%)$ were detected only by the automated system, while $2(2.8 \%)$ were detected only by the conventional method (Refer Table No. 2). McNemar's Chi Square test was done to compare the rate of detection between the two methods. The AutoBCS system had a significantly greater rate of detection than the conventional methods (P value < 0.001) (Refer Table No. 3).

Among the 65 sample pairs with single isolates the automated system detected 63 (96.9\%), while the conventional system detected only 52 (80\%). The automated system had a significantly greater rate of detection than the conventional method even after removing the mixed growth samples from analysis (McNemar's Chi Square P value $<0.01$ ). Early detection of pathogens facilitates initiation of appropriate antibiotic therapy and thus has prognostic significance. On comparison between conventional and automated methods, it was found that the automated system detected $12(17.4 \%)$ cases within 12 hours and 50 cases within 24 hours of incubation, while none was detected by the conventional method within 24 hours (Refer Table No. 4). The mean time of detection of all microorganisms by automated system was 20.88 hours. The gram-negative bacteria had a lower mean time of detection of 18.24 hours compared to 22.8 hours for Gram positive bacteria, but the difference was not statistically significant. Candida which was the sole fungal species isolated had a mean time of detection of 23 hours (Refer Table No. 5). 
Among the organisms isolated by both methods, 53.5 percent were Gram negative organisms and the rest were Gram positive organisms.

Klebsiella pneumoniae, Coagulase Negative Staphylococci and Pseudomonas aeruginosa were the three most common organisms isolated accounting for 13 (18.3\%), 13 (18.3\%) and $11(15.5 \%)$ cases respectively. Candida growth was obtained in $5(7 \%)$ cases, while mixed growth was obtained in $6(8.5 \%)$ cases. All the five Candida growths were detected by both the AutoBCS and conventional systems.

\section{Antibiotic Sensitivity of Gram Positive Bacteria}

Among the total of four isolates of Staphylococcus aureus, two were resistant to Cefoxitin (MRSA). But these isolates were sensitive to Vancomycin, Linezolid, Clindamycin and Rifampicin. Methicillin Sensitive Staphylococcus aureus (MSSA) were sensitive to Gentamicin, first generation Cephalosporin, Erythromycin and Amikacin. All of them were resistant to Penicillin. All MSSA isolates showed sensitivity to Vancomycin, Linezolid, Clindamycin and Rifampicin.

All isolates of Staphylococcus epidermidis were sensitive to Amikacin, Vancomycin, Clindamycin and Rifampicin, while 12 isolates were Cefoxitin resistant.

Of the three Group D Streptococci isolates, two were Enterococcus faecalis and one was an Enterococci other than bovis. Enterococcus faecalis were resistant to Penicillin and were sensitive to Ampicillin. Enterococcus other than bovis was sensitive to both Penicillin and Ampicillin.

All the three Group D Streptococci were sensitive to Vancomycin.

Streptococcus pneumoniae was sensitive to Penicillin, Erythromycin, Cephalosporin and Vancomycin.

\section{Antibiotic Sensitivity of Gram Negative Bacteria}

All the isolates of Enterobacteriaceae showed 100\% sensitivity to Imipenem.

Klebsiella pneumoniae isolates showed maximum sensitivity to Imipenem, Cefoperazone + Sulbactam, Piperacillin - Tazobactam and then to Amikacin.

E. coli isolates also showed similar sensitivity pattern with maximum sensitivity to Imipenem, Amikacin, Cefoperazone-Sulbactam, Piperacillin-Tazobactam and Ciprofloxacin.

The isolates of Pseudomonas aeruginosa were sensitive to Ceftazidime (III generation Cephalosporin), PiperacillinTazobactam and Imipenem; 90.9 percent of Pseudomonas aeruginosa isolates were sensitive to Amikacin and Ciprofloxacin. Only $63.6 \%$ showed sensitivity to Gentamicin.

All the Acinetobacter baumannii isolates were sensitive to Imipenem and Cefoperazone-Sulbactam (100\%). Two isolates were sensitive to Ciprofloxacin. Only $33.33 \%$ of isolates were Amikacin and Piperacillin - Tazobactam sensitive.

Acinetobacter baumannii isolates were $100 \%$ resistant to Ampicillin, I and III generation Cephalosporins.

All the Salmonella typhi and paratyphi isolates were sensitive to Ceftriaxone, Ciprofloxacin and Co-trimoxazole. But among the Paratyphi A, only $66.6 \%$ were sensitive to Chloramphenicol.

Minimum inhibitory concentration of Ciprofloxacin was done along with disc diffusion test.
Flavobacterium meningosepticum isolated was sensitive to Vancomycin, Imipenem, Cefoperazone-Sulbactam and Ciprofloxacin.

\begin{tabular}{|c|c|c|}
\hline & Conventional Method & Automated \\
\hline $\begin{array}{c}\text { Culture } \\
\text { Positive }\end{array}$ & 53 & 69 \\
\hline Sterile & 195 & 179 \\
\hline Total & 248 & 248 \\
\hline Table 1. Overall Rate of Detection in Automated and \\
Conventional Culture Methods \\
\hline
\end{tabular}

\begin{tabular}{|c|c|}
\hline Both Positive & 51 \\
\hline Conventional Alone & 2 \\
\hline Automated Alone & 18 \\
\hline Both Sterile & 177 \\
\hline Total & 248 \\
\hline \multicolumn{2}{|c|}{ Table 2. Culture Positivity of all Sample Pairs } \\
\hline
\end{tabular}

\begin{tabular}{|c|c|c|}
\hline & \multicolumn{2}{|c|}{ Conventional Method } \\
\hline Automated Method & Positive & Sterile \\
\hline Positive & 51 & 18 \\
\hline Sterile & 2 & 177 \\
\hline Table 3. McNemar's Chi Square Test for Significance (P \\
value < 0.001$)$ \\
\hline
\end{tabular}

\begin{tabular}{|c|c|c|c|c|c|c|}
\hline & \multicolumn{3}{|c|}{ Conventional Method } & \multicolumn{3}{|c|}{ Automated Method } \\
\hline Day & Frequency & $\%$ & $\begin{array}{c}\text { Cumulative } \\
\%\end{array}$ & Frequency & $\%$ & $\begin{array}{c}\text { Cumulative } \\
\%\end{array}$ \\
\hline 0 & 0 & 0 & 0 & 50 & 72.5 & 72.5 \\
\hline 1 & 18 & 34 & 34 & 18 & 26.1 & 98.6 \\
\hline 2 & 22 & 41.5 & 75.5 & 1 & 1.4 & 100 \\
\hline 3 & 7 & 13.2 & 88.7 & & & \\
\hline 4 & 5 & 9.4 & 98.1 & & & \\
\hline 5 & 1 & 1.9 & 100 & & & \\
\hline Total & $\mathbf{5 3}$ & $\mathbf{1 0 0}$ & & $\mathbf{6 9}$ & $\mathbf{1 0 0}$ & \\
\hline \multicolumn{7}{|c|}{ Table 4. Comparison of Conventional and Automated } \\
Methods by Day of Detection \\
\hline
\end{tabular}

\begin{tabular}{|c|c|c|c|c|}
\hline & $\mathbf{N}$ & Mean & $\begin{array}{l}\text { Standard } \\
\text { Deviation }\end{array}$ & \\
\hline $\begin{array}{c}\text { Gram } \\
\text { Positive }\end{array}$ & 31 & $\begin{array}{c}0.9955 \text { Days } \\
\text { (23.89 Hours) }\end{array}$ & 0.57 & \multirow{2}{*}{$\begin{array}{c}\text { P-value } \\
0.058\end{array}$} \\
\hline $\begin{array}{c}\text { Gram } \\
\text { Negative }\end{array}$ & 38 & $\begin{array}{c}0.7674 \text { Days } \\
\text { (18.42 Hours) }\end{array}$ & 0.41 & \\
\hline Total & 69 & $\begin{array}{c}\text { 0.8699 Days } \\
\text { (20.88 Hours) }\end{array}$ & 0.5 & \\
\hline
\end{tabular}

\section{DISCUSSION}

The present study is a single centre comparative study for comparing the performance of the newly installed automated blood culture system with the conventional blood culture system. The study took place in the settings of a tertiary care centre, which also acts as a referral centre where timely diagnosis and early initiation of therapy has a prognostic significance. The Microbiology Laboratories play an important role in rapid detection and identification of bacteraemia and initiation of appropriate antibiotic therapy. The performance of the automated blood culture system with respect to rate of detection and time to yield was compared to the conventional method. The study provides an 
opportunity for continuous quality improvement and compliance evaluation to ensure that the potential benefits of newer blood culture technology are optimally utilised.

Positive blood cultures were obtained in 71 (28.6\%) sample pairs, while single isolates were obtained in 65 $(26.2 \%)$ sample pairs in this study. In a study involving suspected cases of neonatal sepsis alone the rate of positivity was 39 percent with automated system and 14 percent with conventional system. 5

The rate of positivity varies from hospital to hospital and the rate of positive cultures would be increased when blood is taken from moribund patients. ${ }^{3}$ The overall positivity also depends on the rate of contamination. The higher than average rate of blood culture positivity obtained can be attributed to the fact that study was conducted in patients with a clinical diagnosis of sepsis or suspected sepsis from the Departments of General Medicine, Infectious Diseases and Paediatrics. Patients from both the wards and intensive care units were also included in this study.

The automated system detected 97.2 percent of the positive samples, while the conventional method detected only 74.7 percent of the positive samples. The automated system had a significantly greater rate of detection than the conventional method even after removing the mixed growth samples from the analysis, which are assumed to be due to contamination. The better performance of automated system in isolation of microbes have been compared with conventional as well as manual continuous systems and found to be superior. ${ }^{6}$ In a comparative study of the paediatric version of automated blood culture system and the conventional method, the automated detected 90 percent of clinically significant isolates compared to 71 percent by the conventional method. 7 In the present study the AutoBCS detected 96.9 percent of the single isolates, while the conventional method detected 80 percent of the single isolates. These findings show that the AutoBCS system has a good isolation performance and is a reliable and better alternative to the conventional system in our settings.

In the present study, the automated system detected 17.4 percent of the positive cases within 12 hours of incubation and 72.5 percent of the cases within 24 hours of incubation. All the positive cases except one sample with a mixed growth were identified within 48 hours of incubation. This is consistent with earlier observations that incubation periods of five days are sufficient for automated blood culture systems and organisms identified beyond five days are usually contaminants. ${ }^{8,9}$ In comparison, the conventional method of blood culture detected none of the cases in the first 24 hours. The conventional method detected 34 percent, 75.5 percent and 88.7 percent of the cases within 48,72 and 96 hours of incubation respectively.

Similar findings are also reported in a study, wherein the AutoBCS performed better by detecting 30 percent of cases within 12 hours and 100 percent of cases within 48 hours. ${ }^{5}$

The mean time for detection of all positive culture was 20.88 hours in the present study. Gram Positive organisms had a slightly longer time for detection when compared to Gram negative organisms. The mean time to detect Candida growth in the present study was 23 hours, which is comparable to other studies.
Horvath et al reported a mean time to growth detection of 25.6 hours for Candida for BacT/ALERT when compared to 27.3 hours for Bactec system. ${ }^{10}$

In the present study as soon as growth was detected by the automated system, a Gram staining was done along with the sub-culture. Gram staining itself could guide empirical therapy to a certain extent. 11 If the Gram staining revealed Gram negative organisms, direct biochemical reactions were also attempted to identify the organisms early to facilitate early reporting.

In the conventional blood culture method, biochemical reactions are usually performed after detecting growth in subcultures. Direct biochemical reactions with the incubated AutoBCS media helped in the identification of organisms and they were confirmed by subsequent tests using the subculture growths. Thus, the detection and identification of pathogens, especially Gram negative bacteria could be advanced by as much as 24 hours. This helps the clinicians to initiate appropriate antibiotics as early as possible.

In addition, the alarm system in the AutoBCS facilitates early sub-culture of the specimen, thus facilitating early antibiotic sensitivity testing and reporting. This is in contrast to the conventional system, where the initial subculture is done after six hours and then repeated every 24 hours. This is especially useful in facilities with less number of staff to monitor the daily load of blood cultures. The work load is considerably reduced as the laboratory staff does not have to do subcultures in all the cases. ${ }^{3}$

Hardy et al found a 0.2 percent positivity among terminal subculture of negative AutoBCS bottles. ${ }^{8}$ Another study from Korea, which evaluated the negative AutoBCS results using terminal sub-cultures found 2.6 percent of the sub-cultures to be positive. ${ }^{12}$ Automated systems are also good for culturing other sterile fluids. 13

In the present study 53.5 percent isolates were Gram negative organisms and 46.5 percent were Gram positive organisms. Klebsiella pneumoniae, Coagulase negative Staphylococci and Pseudomonas aeruginosa were the three most common organisms isolated accounting for $13(18.3 \%)$, $13(18.3 \%)$ and $11(15.5 \%)$ cases respectively. Candida growth was obtained in $5(7 \%)$ cases, while mixed growth was obtained in $6(8.5 \%)$ cases. All the five Candida growth were detected by both the AutoBCS and conventional systems.

Although automated continuous-monitoring blood culture systems are both rapid and sensitive, false-positive and false-negative results still occur. In the present study, the conventional method detected two cases of Coagulase negative staphylococci that were not detected by the AutoBCS. They were most likely to be contaminants, as they were also not detected by terminal sub-cultures from the AutoBCS bottles. ${ }^{14}$

A sentinel surveillance study showed that infections due to Gram-negative organisms are increasing and E. coli was the most common organism isolated.15,16 Present study showed Klebsiella pneumonia followed by Pseudomonas aeruginosa as commonest Gram negative bacilli isolated. Nosocomial infections with multidrug resistant strains are increasing nowadays. $17,18,19,20$

Even though the study was focussing on bacterial pathogens, Candida isolates were also obtained and were the 
fourth most common cause of blood infections. Three of the patients were given antifungal treatment. ${ }^{21}$

\section{CONCLUSION}

The present study was a comparative study on conventional and automated blood culture system with respect to rate and time of detection of blood culture. About 78 percentage positive samples were detected by AutoBCS in first twenty four hours, which enabled early processing and reporting. Rate of detection of the AutoBCS was also significant when compared to conventional. Specimen handling could be reduced by AutoBCS. The mean time for detection by the automated method was 22.8, 18.24 and 23 hours for Gram positive bacteria, Gram negative bacteria and fungi respectively. They can be a valuable tool for the early detection and identification of blood pathogens. This improves the prognosis of those patients admitted with fever and/or sepsis. The rapid and reliable detection of blood stream infections helps in timely initiation of appropriate antibiotics. Automated blood culture systems are a reliable alternative to conventional blood culture systems.

\section{REFERENCES}

[1] Weinstein MP. Current blood culture methods and systems: clinical concepts, technology and interpretation of results. Clin Infect Dis 1996;23(1):40-6.

[2] Ntusi N, Aubin L, Oliver S, et al. Guideline for the optimal use of blood cultures. S Afr Med J 2010;100(12):839-43.

[3] Gould JC, Duerden BI. Symposium report blood culture--current state and future prospects. Abstracts. J Clin Pathol 1983;36(9):963-77.

[4] Bryan CS. Clinical implications of positive blood cultures. Clin Microbiol Rev 1989;2(4):329-53.

[5] Maria A, Phalgun S, Hans C, et al. Comparison of conventional blood culture method and BacT/Alert system with respect to yield and time to positivity in neonatal sepsis. Early Human Development 2011;87:S89.

[6] Rohner P, Pepey B, Auckenthaler R. Comparison of BacT/Alert with Signal blood culture system. J Clin Microbiol 1995;33(2):313-7.

[7] Krisher KK, Whyburn DR, Koepnick FE. Comparison of the BacT/Alert pediatric blood culture system, PediBacT, with conventional culture using the 20 -milliliter Becton-Dickinson supplemented peptone broth tube. J Clin Microbiol 1993;31(4):793-7.

[8] Hardy DJ, Hulbert BB, Migneault PC. Time to detection of positive BacT/Alert blood cultures and lack of need for routine subculture of 5- to 7-day negative cultures. J Clin Microbiol 1992;30(10):2743-5.

[9] Wilson ML, Mirrett S, Reller LB, et al. Recovery of clinically important microorganisms from the BacT/Alert blood culture system does not require testing for seven days. Diagn Microbiol Infect Dis 1993;16(1):31-4.
[10] Horvath LL, George BJ, Murray CK, et al. Direct comparison of the BACTEC 9240 and BacT/ALERT 3D automated blood culture systems for candida growth detection. Journal of Clinical Microbiology 2004;42(1):115-8.

[11] Barenfanger J, Graham DR, Kolluri L, et al. Decreased mortality associated with prompt Gram staining of blood cultures. Am J Clin Pathol 2008;130(6):870-6.

[12] Kocoglu ME, Bayram A, Balci I. Evaluation of negative results of BacT/Alert 3D automated blood culture system. J Microbiol 2005;43(3):257-9.

[13] Lakshmi V. Culture of body fluids using the bact/alert system. Indian Journal of Medical Microbiology 2001;19(2):44-50.

[14] Struthers S, Underhill H, Albersheim S, et al. A comparison of two versus one blood culture in the diagnosis and treatment of Coagulase Negative Staphylococcus in the neonatal intensive care unit. J Perinatol 2002;22(7):547-9.

[15] Weinstein MP, Murphy JR, Reller LB, et al. The clinical significance of positive blood cultures: a comprehensive analysis of 500 episodes of bacteremia and fungemia in adults. II. Clinical observations, with special reference to factors influencing prognosis. Rev Infect Dis 1983;5(1):54-70.

[16] Diekema DJ, Pfaller MA, Jones RN, et al. Survey of bloodstream infections due to Gram-negative bacilli: frequency of occurrence and antimicrobial susceptibility of isolates collected in the United States, Canada, and Latin America for the SENTRY Antimicrobial Surveillance ProGram, 1997. Clin Infect Dis 1999;29(3):595-607.

[17] de la Torre MG, Romero-Vivas J, Martinez-Beltran J, et al. Klebsiella bacteremia: an analysis of 100 episodes. Rev Infect Dis 1985;7(2):143-50.

[18] Benjamin DK, DeLong E, Cotten CM, et al. Mortality following blood culture in premature infants: increased with Gramnegative bacteremia and candidemia, but not Gram-positive bacteremia. J Perinatol 2004;24(3):175-80.

[19] Kennedy KJ, Roberts JL, Collignon PJ. Escherichia coli bacteraemia in Canberra: incidence and clinical features. Med J Aust 2008;188(4):209-13.

[20] Navon-Venezia S, Ben-Ami R, Carmeli Y. Update on Pseudomonas aeruginosa and Acinetobacter baumannii infections in the healthcare setting. Curr Opin Infect Dis 2005;18(4):306-13.

[21] Morrell M, Fraser VJ, Kollef MH. Delaying the empiric treatment of candida bloodstream infection until positive blood culture results are obtained: a potential risk factor for hospital mortality. Antimicrob Agents Chemother 2005;49(9):3640-5. 